\title{
The prevalence of alloantibody in the population of Xinjiang area of China
}

\author{
Limin Zhang ${ }^{1}$, Xiao E ${ }^{2}$, Haiying Wang ${ }^{1}$, Mingjun Huang ${ }^{3}$, Gulinuer Kuerban ${ }^{1}$, Xiaoli Sun ${ }^{1}$, \\ Senru Zhang ${ }^{1}$, Yanchao Xing ${ }^{1 *}$ \\ ${ }^{1}$ Department of Transfusion, The General Hospital of Xinjiang Military Command of PLA, Urumqi, Xinjiang, 830000, China; \\ ${ }^{2}$ Department of Pharmacy, The General Hospital of Xinjiang Military Command of PLA, Urumqi, Xinjiang, 830000, China; \\ ${ }^{3}$ The Medicial Unit of Kizilsu Military Sub-District of Xinjiang Military Command of PLA, Kizilsu Kirghiz Autonomous \\ Prefecture, Xinjiang, 845350, China
}

\begin{abstract}
Alloantibodies are the major cause of hemolytic transfusion reaction and newborn hemolytic disease. It is highly recommended to screen alloantibodies before transfusion and pregnancy. This report applied the microcolum gel method to screen for available alloantibodies, enrolling 20,098 patients from January 2016 to December 2017 in the Xinjiang General Hospital. Seventy-two patients were found alloantibody-positive, at an overall positive rate of $0.35 \%$. The distribution of alloantibody varied according to age, gender or treatment. The patients aged 70 plus and the patients admitted in the Department of Hematology and Department of Gynecology \& Obstetrics had a higher incidence rate of alloantibodies. By using 10 screening cell panel systems, 9 types of alloantibodies including anti-D, anti-E, anti-e, anti-C, anti-c, anti-M, anti-s, anti-Fy ${ }^{\mathrm{b}}$ and anti-Ce were identified. This study suggests that the transfusion of red blood cells(RBCs) and pregnancy are the main causes of alloantibodies.
\end{abstract}

Keywords: alloantibody, transfusion, pregnancy

\section{INTRODUCTION}

An alloantibody is a blood group antibody found in plasma, which often has important consequences for blood transfusion and pregnancy outcome. An alloantibody may cause an incompatibility in the cross match test and lead to a delay in transfusion, which in turn may threaten patients' lives under urgent conditions. The IgG type alloantibody for example is sometimes able to penetrate placental barrier and induce hemolytic disease of the newborn. Therefore, it is recommended to screen alloantibodies before blood transfusion or labor. In this paper, the alloantibodies were retrospectively analyzed in 20,098 patients from Xinjiang General Hospital, and the main causes of al- loantibodies were investigated.

\section{MATERIALS AND METHODS}

\section{Sample source and screening of alloantibody}

The patient's information including gender, age and department admitted was collected and 20,098 patients were screened the irregular antibody. A $2-3 \mathrm{~mL}$ venous blood sample was collected from each subject in EDTA anticoagulated vials, from which plasma was separated. All the samples were screened for irregular antibody using the microcolum gel method, with three cell panels. The direct agglutination test (DAT) and auto control (AC) were also performed to exclude the influence of autoantibody. The alloantibody-positive

*Correspondence to: Yanchao Xing, Department of Transfusion, The General Hospital of Xinjiang Military Command of PLA, Urumqi, Xinjiang, 830000, China.E-mail: xingyanchao@aliyun.com.

Conflict of interests: The authors have declared no conflict of interests. 
samples were subsequently screened for alloantibody identification by microcolum gel method, using ten cell panels. The microcolum gel cards were purchased from Bio-Rad(USA), and the panel cells, screening cells and AHG-anti IgG were from Shanghai Blood Biomedical Co., Ltd.(China).

This research was approved by the Ethics Committee Board of our hospital, and the patients signed the document of informed consent.

\section{Statistical analysis}

The statistical analysis was performed with SPSS, and $P<0.05$ was considered statistically significant.

\section{RESULTS}

\section{The incidence rate of alloantibody in different groups}

In 20,098 patients, 72 alloantibody-positive cases were diagnosed, and the incidence rate was $0.35 \%$. In the 72 positive cases, 46 cases were female, and 26 cases were male. The incidence rate increased with age, and the 70 plus age group had the largest proportion (Table 1).

Table 1 Positive cases in different age group

\begin{tabular}{ccc}
\hline Age group & Positive cases & Proportion $(\%)$ \\
\hline $0-30$ y & 8 & 11.11 \\
$31-40$ y & 8 & 11.11 \\
$41-50$ y & 13 & 18.06 \\
$51-60$ y & 11 & 15.28 \\
$61-70$ y & 12 & 16.67 \\
$70+$ y & 20 & $27.77^{*}$ \\
\hline
\end{tabular}

${ }^{*} P<0.05$ compared with other age group.

\section{The incidence rate of alloantibody in different departments}

The incidence rate of alloantibody in the Department of Internal Medicine (excluding the Department of Hematology), the Department of Hematology, the Department of Surgery (excluding the Department of Gynecology and Obstetrics) and the Department of Gynecology and Obstetrics were $0.327 \%, 0.998 \%$, $0.286 \%$, and $0.385 \%$, respectively (Table 2 ).

\section{The frequency of different alloantibodies}

Alloantibody identification was performed and 9

Table 2 Alloantibody-positive cases in different department

\begin{tabular}{|c|c|c|c|}
\hline Department & $\begin{array}{l}\text { Screened } \\
\text { cases }\end{array}$ & $\begin{array}{c}\text { Positive } \\
\text { cases }\end{array}$ & $\begin{array}{r}\text { Positive } \\
\operatorname{rate}(\%)\end{array}$ \\
\hline Internal Medicine & 6,733 & 22 & 0.327 \\
\hline Hematology & 1,403 & 14 & $0.998^{*}$ \\
\hline Surgery & 10,143 & 29 & 0.286 \\
\hline Gynecology \& Obstetrics & 1,819 & 7 & $0.385^{\#}$ \\
\hline
\end{tabular}

types of alloantibody were identified. The frequency of each alloantibody was shown in Table 3. Anti-E $(0.089 \%)$, anti-c $(0.059 \%)$ and anti-M $(0.054 \%)$ were more frequent than other alloantibodies.

Table 3 Frequency of alloantibodies

\begin{tabular}{lrc}
\hline Alloantibody & Cases & Frequency $(\%)$ \\
\hline Ant-D & 1 & 0.005 \\
Anti-E & 18 & 0.089 \\
Anti-e & 5 & 0.025 \\
Anti-C & 6 & 0.029 \\
Anti-c & 12 & 0.059 \\
Anti-M & 11 & 0.054 \\
Anti-s & 8 & 0.039 \\
Anti-Fy $^{\mathrm{b}}$ & 9 & 0.044 \\
Anti-C,-e & 2 & 0.010 \\
\hline
\end{tabular}

\section{DISCUSSION}

The prevalence of alloantibody varies in different areas and populations. Makroo et al. reported that the positive rate of alloantibody was $0.29 \%$ in healthy blood donors in India ${ }^{[1]}$. Another report by Takeshita et al. found that the prevalence of alloantibody of patient was higher, at $1.43 \%$ in Japan ${ }^{[2]}$. A systematic literature review reported that the incidence rate of unexpected red blood cell antibodies was $0.23 \%$ in China ${ }^{[3]}$, and that there are differences among the different areas of China. For example, Duan et al. reported the prevalence of alloantibodies in healthy blood donors was $1.23 \%$ in the city of Hengyang in Hunan province ${ }^{[4]}$, while Tang et al. reported that the incidence rate of irregular antibodies in patients was $0.4 \%$ in the Nanjing area ${ }^{[5]}$. In this report, the patients in Xinjiang General Hospital were screened from January 2016 to December 2017. Alloantibody was positive in 72 cases, with an overall incidence rate of $0.35 \%(72 / 20,098)$. The incidence rates in different regions of China were listed in Table 4, which indicated that Xinjiang had the lowest incidence rate $(0.35 \%)$, which may be due to different racial characteristics.

During pregnancy, females are exposed to allo -antigen more frequently, and subsequently have a higher possibility of being alloantibody positive. Weinstein reported the alloantibody incidence rate in the Caucasian pregnant female population was $1 \%-$ $2 \%{ }^{[6]}$, and Lee et al. reported that the prevalence of irregular antibody was $0.64 \%$ in pregnant Han Chinese individuals ${ }^{[7]}$. In this paper, our data suggested that in alloantibody-positive cases, females account for $63.89 \%$, which was significantly higher than that of males $(36.11 \%)$. Our data showed that the incidence rate of alloantibody increased with age, and the 70 plus age group accounted for the most of the positive 
Table 4 The prevalence of alloantibodies in different region of China

\begin{tabular}{lccc}
\hline Region & Screened cases & Positive cases & Incidence rate (\%) \\
\hline Shaanxi $^{[8]}$ & 79,403 & 409 & 0.51 \\
Jilin $^{[9]}$ & 1,825 & 12 & 0.66 \\
Shenzhen $^{[10]}$ & 5,628 & 40 & 0.71 \\
Beijing $^{[11]}$ & 9,013 & 57 & 0.63 \\
Ningxia $^{[12]}$ & 2,572 & 18 & 0.70 \\
Jiangsu $^{[5]}$ & 29,770 & 120 & 0.40 \\
Henan $^{[13]}$ & 12,693 & 84 & 0.66 \\
Hunan $^{[14]}$ & 24,743 & 138 & 0.56 \\
Jilin $^{[15]}$ & 10,000 & 78 & 0.78 \\
Fujian $^{[16]}$ & 3,025 & 32 & 1.10 \\
\hline
\end{tabular}

cases. This might be caused by the higher accessibility of alloantigen in the aged population.

The incidence rates of alloantibody were distributed in different departments unequally. There was no significant difference between the Internal Medicine Department and Surgery Department. However, the alloantibody prevalence rate was $0.998 \%$ in the patients of the Hematology Department which is higher than that of other Departments of Internal Medicine, and $0.385 \%$ in the patients of the Department of Gynaecology \& Obstetrics which is higher than that of other Departments of Surgery, respectively. In order to alleviate anemia symptoms, the patients in Department of Hematology require frequent transfusions of red blood cells(RBCs), so they have a greater chance of contacting alloantigen. In some cases involving pregnant women, the fetus' RBC may penetrate the placental barrier and stimulate the occurrence of alloantibody in the mother. These facts most likely explain the high prevalence of alloantibody in patients of the Department of Hematology and the Department of Gynecology \& Obstetrics. Using the ten cell panel system, nine types of alloantibody were identified. Among the nine types of alloantibodies, the incidence rates of anti-E, anti-c and anti-M were $0.089 \%, 0.059 \%$ and $0.054 \%$ respectively, which were higher than those of other types of alloantibodies.

Due to ethnical differences, the incidence of alloantibody in Xinjiang was lower than those in other regions of China. This study highlights the need of routine screening for alloantibodies among multi-transfused patients and pregnant females. The antibody screening of routine pre-transfusion and pre-labor is essential to ensure safer transfusions and reduce the risk of hemolytic disease of the newborn.

\section{References}

[1] Makroo RN, Rajput S, Agarwal S, et al. Prevalence of irregular red cell antibody in healthy blood donors attending a tertiary care hospital in North India. Asian $J$ Transfus Sci, 2018, 12(1):17-20.

[2] Takeshita A, Watanabe H, Fijihara H, et al. Collaborative study of irregular erythrocyte antibodies in Japan: results from the Japanese study group of allo-immunity and antigen diversity in Asian populations. Transfus Apher Sci, 2010, 43(1): 3-8.

[3] Chen C, Tan J, Wang L, et al. Unexpected red blood cell antibody distributions in Chinese people by a systematic literature review. Transfusion, 2016, 56(4): 975-9.

[4] Duan R, Guo J, Wang X, The clinical value of irregular antibody screening in clinical safe blood transfusion in 17,652 healthy blood donors. China Modern Doctor(in Chinese), 2017, 55(17):85-7.

[5] Tang W, Zhu PY, Yan JM, et al. Preliminary investigation and analysis of irregular antibodies of blood group before patients transfusion. Journal of Medical Postgraduates(in Chinese), 2016,29(3):272-5.

[6] Weinstein L. Irregular antibodies causing hemolytic disease of the newborn: a continuing problem. Clin Obstet Gynecol, 1982,25(2):321-32.

[7] Lee CK, Ma ESK, Tang M, et al. Prevalence and specificity of clinically significant red cell alloantibodies in Chinese women during pregnancy-a review of cases from 1997 to 2001. Transfus Med, 2003,13(4):227-31.

(Received 12 December 2018, Revised 10 February 2019, Accepted 03 March 2019) 


\section{Piperacillin Antibody Detective Kit (Micro-column Gel Test)}

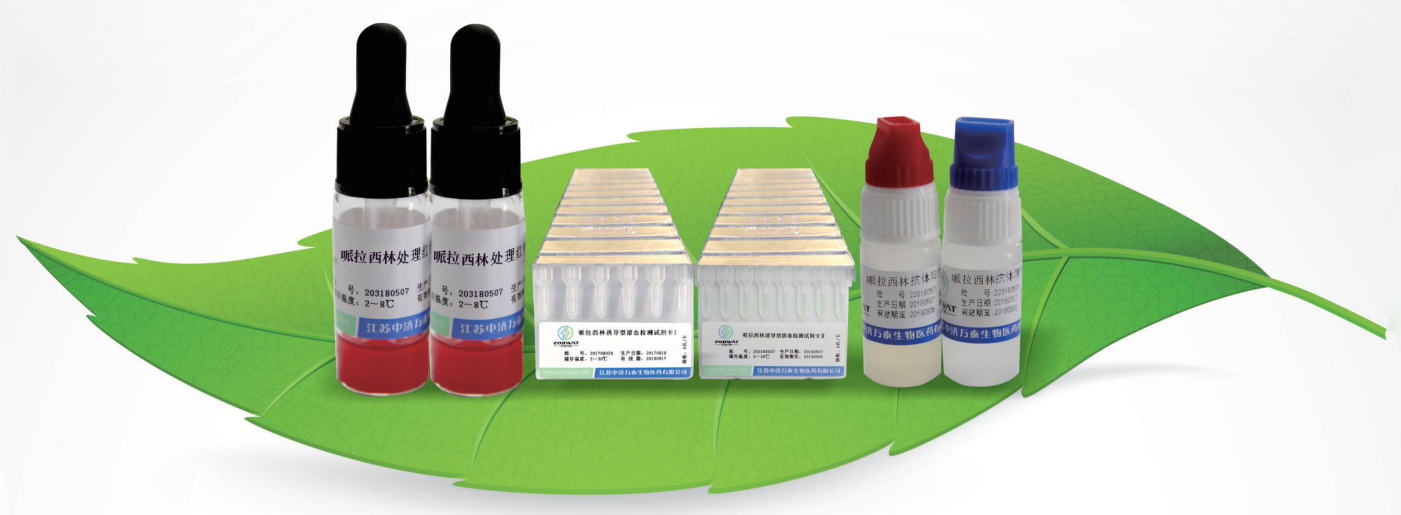

【Description and Specification】

Description

Piperacillin Antibody Examination Kit I

Piperacillin Antibody Examination Kit II

Piperacillin Sensitized RBC

Control RBC

Positive Control

Negative Control

\section{Specification}

6 well / card $x 12$ card / box

8 well / card $x 12$ card / box

$2.5 \mathrm{ml} /$ vial $x 1$ vial / box

$1 \mathrm{ml} /$ vial $x 1$ vial / box

$1.5 \mathrm{ml} /$ vial $\mathrm{x} 1$ vial / box

$1.5 \mathrm{ml} / \mathrm{vial} \times 1 \mathrm{vial} / \mathrm{box}$

Storage Temp. : $2 \sim 8^{\circ} \mathrm{C}$ Expiration Date: 3 months

\section{【Packing Specifications】24T/box}

\section{【Application of Product】}

ZJWT Piperacillin Antibody Detective Kit is intended for the qualitative detection of the Piperacillin Antibody from plasma through drug coating red blood cell, to guide medication of antibiotics. Patient use only, not for blood donor examination.

\section{Safeguard of Medication Decision}

JiangSu ZoJiWat Bio-Pharmaceutical Co.,Ltd.

Address: No. 78 West Dongsheng Road, Jiangyin, Jiangsu214400 , P,R.China

Tel: 0510-81695091, 81695092

Fax: 0510-81695129

Http: www.jszojiwat.com
Sales specialist: Mr. Zhang Mobile:15370255367

Mail:zhangyuchuan@jszojiwat.com

Technical services: 15190368370

Mail: caochen@jszojiwat.com

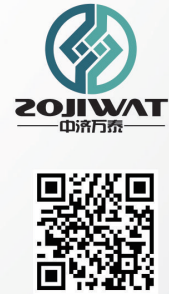

